\title{
Ipilimumab Associated Autoimmune Alveolitis Responding to Corticosteroids
}

\author{
Ankit R. Rao ${ }^{\mathrm{a}, \mathrm{b}}$, Simon A. Grumett
}

\begin{abstract}
Malignant melanoma is increasing in incidence and metastatic melanoma is extremely challenging to treat, although new targeted and immunotherapeutic options for treatment are emerging. One of these is the anti CTLA4 antibody ipilimumab which releases the 'break' on endogenous anti-melanoma T-cell responses leading to improved survival. It is associated with autoimmune toxicity, typically in the liver, skin and colon. We report here the case of an ipilimumab treated patient who developed autoimmune alveolitis due to the drug, which is only very rarely reported in association with this form of immune-modulating therapy.
\end{abstract}

Keywords: Ipilimumab; Melanoma; Alveolitis

\section{Introduction}

Approximately $30 \%$ of all patients with malignant melanoma are found to have distant metastatic disease whether at presentation or at a later point. Metastatic melanoma is thought to be relatively insensitive to standard approaches such as cytotoxic chemotherapy [1] and radiotherapy. However, it has long been known that anti-melanoma immune responses can develop and occasionally retard the progression of the disease [2]. Immunotherapy with cytokines such as interferon alpha and interleukin-2 has some efficacy in the adjuvant and metastatic settings respectively [3, 4]. More recently ipilimumab, a fully humanised monoclonal antibody that antagonises human CTLA4, has shown a definite survival benefit in metastatic melanoma despite relatively

\footnotetext{
Manuscript accepted for publication September 24, 2012

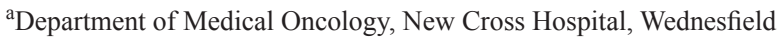
Road, Wolverhampton, United Kingdom

${ }^{\mathrm{b}}$ Corresponding author: Ankit R. Rao, New Cross Hospital, Wednesfield Road, Wolverhampton, U.K.

Email: ankitrao@doctors.org.uk
}

doi:http://dx.doi.org/10.4021/jmc886w modest objective response rates [5]. Human CTLA4 is expressed on T-lymphocytes and is upregulated on T-cell activation: it functions as a 'brake' on the immune system [6]. Ipilimumab permits sustained activation of anti-melanoma adaptive responses. Its toxicity profile is quite different to that of conventional chemotherapy and includes potentially life threatening autoimmunity in any organ system [7]. Here we present a case of ipilimumab-associated pneumonitis which has only been very rarely reported.

\section{Case Report}

A 57-year-old Caucasian man was diagnosed with metastatic melanoma with multiple bilobar hepatic metastases and a left axillary matted nodal mass (T4a N3 M1c). He had previously had resection of a $6 \mathrm{~mm}$ cutaneous melanoma. The interval from primary surgery to development of metastatic disease was 9 months. His tumour did not carry any mutations in the B-raf gene. He was initially treated with intravenous dacarbazine ( $1 \mathrm{~g} / \mathrm{m}^{2} 3$ weekly) but a CT scan after 2 cycles of chemotherapy showed a mixed response with slight reduction in size of the left axillary mass but progression of the liver metastases. Therefore, he was considered for immune therapy with ipilimumab. There was no personal or family history of autoimmune disorders and he had no known endocrine disturbance. After the first treatment with ipilimumab at a dose of $3 \mathrm{mg} / \mathrm{kg}$ intravenously, he developed a Grade 1 skin reaction but no other signs of toxicity. However, nine days after the second infusion he presented with marked breathlessness on minimal exertion, a non-productive cough and a Grade 2-3 generalised erythematous skin rash. Clinical examination revealed tachypnea, tachycardia and extensive inspiratory crackles throughout both lung fields with no wheeze. The patient was afebrile and normotensive but oxygen saturations were $90 \%$ breathing room air. Arterial blood gas analysis confirmed hypoxaemia with a $\mathrm{PaO} 2$ of $6.84 \mathrm{kPa}$. Chest radiograph showed bilateral patchy lower zone infiltrates (Fig. 1) and laboratory investigations were remarkable only for a modestly elevated C-reactive protein $(58 \mathrm{mg} / \mathrm{L} \mathrm{NR}$ 0 - 6), very slight lymphopaenia $\left(1.4 \times 10^{9} / \mathrm{mL}\right.$ NR $\left.1.5-4\right)$ and a moderate eosinophilia $\left(1.3 \times 10^{9} / \mathrm{L}\right.$ NR $\left.0-0.4\right)$. He was 


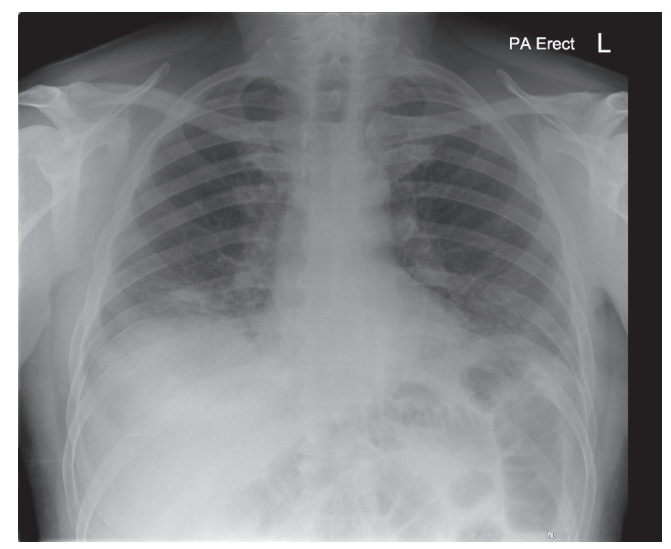

Figure 1. Chest Radiograph on admission: bilateral lower zone infiltrates

treated with high dose corticosteroids orally (dexamethasone $8 \mathrm{mg}$ twice daily) along with oral antibiotics (clarithromycin $500 \mathrm{mg}$ twice daily) for the first 36 hours only and continuous oxygen. CT scan after 48 hours demonstrated bilateral ground glass and reticulonodular changes consistent with acute alveolitis bilaterally (Fig. 2) particularly in the lower zones with no evidence of pulmonary thromboembolism. The imaged liver showed a deterioration in appearances of the hepatic metastases since the initiation of ipilimumab. There was however, a dramatic improvement in his cough and breathlessness and he did not require supplemental oxygen by 72 hours of admission. There was resolution of the bilateral infiltrates on chest radiograph (Fig. 3) by day 5 after admission. His corticosteroids were reduced and stopped within 14 days. However, a week after the third treatment with ipilimumab, mild breathlessness on exertion returned with a non-productive cough and dexamethasone was reinstituted with good effect. The patient has completed 4 cycles

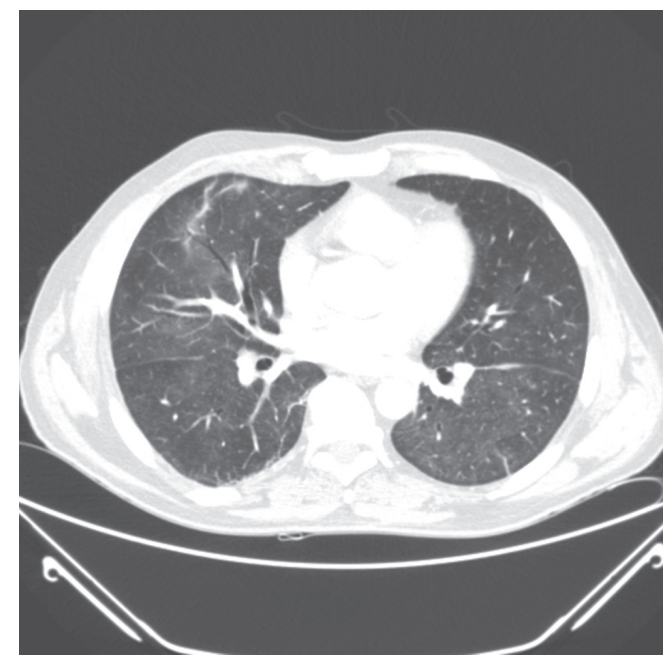

Figure 2. CT thorax demonstrating ground glass shadowing bilaterally

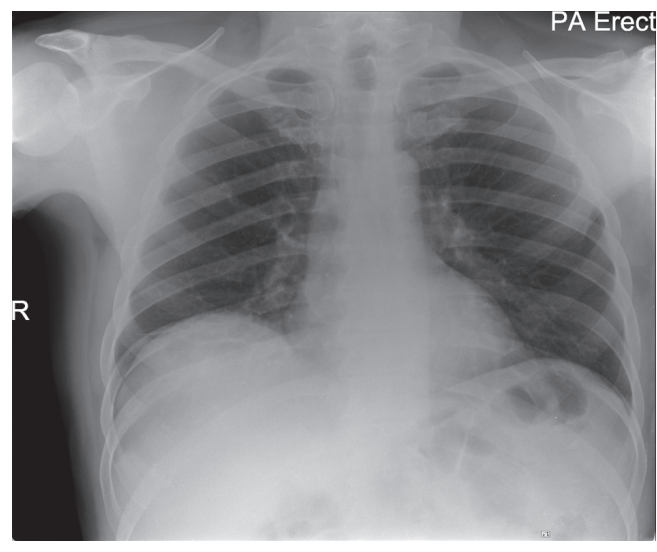

Figure 3. Chest Radiograph taken 5 days after commencement of corticosteroids: resolution

of induction ipilimumab without further complications and is currently off steroid treatment.

\section{Discussion}

Ipilimumab is a fully humanised monoclonal antibody directed against human CTLA4 (cytotoxic T lymphocyte antigen 4). CTLA4 is upregulated on activated T-lymphocytes and competes with $\mathrm{CD} 28$ for binding to costimulatory molecules B7.1 and B7.2 [8]. Its interaction with B7 molecules leads to downregulation of T-cell activation, increased apoptosis and acts as a negative regulator of T-cell function [6]. Therapeutic CTLA4 blockade is thought to enhance T-cell priming which can lead to augmentation of anti-tumour Tcell responses [9]. Ipilimumab is an effective second-line treatment, after chemotherapy or cytokine therapy, for advanced melanoma as evidenced by increased 2 year survival over a gp100 peptide vaccine approach. The drug also has activity and survival benefit in the first line setting for the same disease in combination with standard dacarbazine chemotherapy, although discontinuation rates were high in the combination therapy arm [10]. As expected from the mechanism of action, and in light of the experimental evidence that transgenic animals lacking CTLA4 display overwhelming and often fatal autoimmunity [11], it is unsurprising that this drug is associated with a range of immune-related adverse events. Grade 3-4 adverse events occur in $10-20 \%$ of patients in randomised trials. These can be divided into early and delayed effects. The most common acute IRAEs include colitis, dermatitis and hepatitis with dermatitis occurring as early as one dose of drug. Autoimmune adverse effects have been correlated with clinical benefit from the drug [12] and are often associated with a dense mixed lymphocytic (CD8 and CD4 T-cells) and neutrophilic infiltrate into the organ involved [13]. Delayed autoimmunity tends to affect endocrine glands including the pituitary, the adrenal cortex 
and the thyroid. Rare targets for autoimmunity include the joints and kidneys [14]. In Phase II studies less than 1 in 100 treated patients developed Grade 3 or 4 cough, dyspnea or pleural effusion [15]. There is only one previous report of autoimmune pulmonary toxicity related to ipilimumab administration [16]. The adverse pulmonary events, dyspnea and pneumonitis, occurred in patients treated with ipilimumab who had relapsed haematologic malignancies after allogeneic haematopoetic stem cell transplantation. In this context, ipilimumab was being used to enhance responses of allogeneic T-lymphocytes against host tumour cells (graft versus leukaemia effect). In one patient with relapsed Hodgkin's lymphoma, breathlessness with an obstructive deficit on spirometry but a normal CT scan of the thorax developed 10 weeks after ipilimumab at a dose of $3 \mathrm{mg} / \mathrm{kg}$. In the other patient, again with Hodgkin's lymphoma treated at $3 \mathrm{mg} / \mathrm{kg}$, there was an initial clinical and radiological response followed by disease progression. On ipilimumab re-challenge, bilateral upper lung lobe ground-glass opacities were noted but the patient was asymptomatic. Transbronchial biopsy showed inflammatory changes and microbiological studies were negative. There was no response to antimicrobials and the infiltrates worsened before responding dramatically to corticosteroid therapy. However, there was recrudescence of infiltrates and marked dyspnea requiring intubation on cessation of corticosteroids. This was treated with infliximab and corticosteroids, and a subsequent recurrence of autoimmune alveolitis required a combination of infliximab, corticosteroids and mycophenolate mofetil. Pneumonitis due to ipilimumab in patients with solid cancers has not been reported. In our case, we believe the diagnosis was ipilimumab induced autoimmune alveolitis/pneumonitis in view of the dry, non-productive cough, alveolar ground-glass changes bilaterally on CT scan, normal temperature, and modestly elevated CRP. The excellent symptomatic improvement with high-dose corticosteroid treatment, but only 36 hours of antibiotics, also supports this. Perhaps the most suggestive evidence that an autoimmune process in the lungs had been released by the drug was that there was a simultaneous marked increase in size and number of liver metastases on CT scan likely due to immune infiltration of hepatic deposits. Our case is distinguished by the simultaneous occurrence of grade 3 dermatologic and pulmonary toxicity. This case highlights the importance of the patient and health-care professionals being fully informed of the possibility of severe autoimmunity developing in any organ system, so that corticosteroids can be initiated promptly and also the need to make a baseline assessment of patients' pulmonary reserve and comorbidity prior to ipilimumab initiation.

\section{References}

1. Serrone L, Hersey P. The chemoresistance of hu- man malignant melanoma: an update. Melanoma Res. 1999;9(1):51-58.

2. Sahin U, Tureci O, Schmitt H, Cochlovius B, Johannes T, Schmits R, Stenner F, et al. Human neoplasms elicit multiple specific immune responses in the autologous host. Proc Natl Acad Sci U S A. 1995;92(25):1181011813.

3. Kirkwood JM, Strawderman MH, Ernstoff MS, Smith TJ, Borden EC, Blum RH. Interferon alfa-2b adjuvant therapy of high-risk resected cutaneous melanoma: the Eastern Cooperative Oncology Group Trial EST 1684. J Clin Oncol. 1996;14(1):7-17.

4. Atkins MB, Lotze MT, Dutcher JP, Fisher RI, Weiss G, Margolin K, Abrams J, et al. High-dose recombinant interleukin 2 therapy for patients with metastatic melanoma: analysis of 270 patients treated between 1985 and 1993. J Clin Oncol. 1999;17(7):2105-2116.

5. Hodi FS, O'Day SJ, McDermott DF, Weber RW, Sosman JA, Haanen JB, Gonzalez R, et al. Improved survival with ipilimumab in patients with metastatic melanoma. N Engl J Med. 2010;363(8):711-723.

6. Gribben JG, Freeman GJ, Boussiotis VA, Rennert P, Jellis CL, Greenfield E, Barber M, et al. CTLA4 mediates antigen-specific apoptosis of human $\mathrm{T}$ cells. Proc Natl Acad Sci U S A. 1995;92(3):811-815.

7. Di Giacomo AM, Biagioli M, Maio M. The emerging toxicity profiles of anti-CTLA-4 antibodies across clinical indications. Semin Oncol. 2010;37(5):499-507.

8. Perez VL, Van Parijs L, Biuckians A, Zheng XX, Strom $\mathrm{TB}, \mathrm{Abbas} \mathrm{AK}$. Induction of peripheral T cell tolerance in vivo requires CTLA-4 engagement. Immunity. 1997;6(4):411-417.

9. Leach DR, Krummel MF, Allison JP. Enhancement of antitumor immunity by CTLA-4 blockade. Science. 1996;271(5256):1734-1736.

10. Robert C, Thomas L, Bondarenko I, O’Day S, M DJ, Garbe $\mathrm{C}$, Lebbe $\mathrm{C}$, et al. Ipilimumab plus dacarbazine for previously untreated metastatic melanoma. N Engl J Med. 2011;364(26):2517-2526.

11. Tivol EA, Borriello F, Schweitzer AN, Lynch WP, Bluestone JA, Sharpe AH. Loss of CTLA-4 leads to massive lymphoproliferation and fatal multiorgan tissue destruction, revealing a critical negative regulatory role of CTLA-4. Immunity. 1995;3(5):541-547.

12. Attia P, Phan GQ, Maker AV, Robinson MR, Quezado MM, Yang JC, Sherry RM, et al. Autoimmunity correlates with tumor regression in patients with metastatic melanoma treated with anti-cytotoxic T-lymphocyte antigen-4. J Clin Oncol. 2005;23(25):6043-6053.

13. Kleiner DE, Berman D. Pathologic changes in ipilimumab-related hepatitis in patients with metastatic melanoma. Dig Dis Sci. 2012;57(8):2233-2240.

14. Sammartino, Goodman, Flanagan et al. Anti GBM disease following CTLA4 blockade in a patient 
with metastatic melanoma. Clinical Kidney Journal. 2010;3(2):135-137.

15. O’Day SJ, Maio M, Chiarion-Sileni V, Gajewski TF, Pehamberger H, Bondarenko IN, Queirolo P, et al. Efficacy and safety of ipilimumab monotherapy in patients with pretreated advanced melanoma: a multicenter single- arm phase II study. Ann Oncol. 2010;21(8):1712-1717.

16. Bashey A, Medina B, Corringham S, Pasek M, Carrier E, Vrooman L, Lowy I, et al. CTLA4 blockade with ipilimumab to treat relapse of malignancy after allogeneic hematopoietic cell transplantation. Blood. 2009;113(7):1581-1588. 